\title{
LOW LEVEL LASER THERAPY: HEALING AT THE SPEED OF LIGHT
}

Samith Ahmed ${ }^{1}$, Gregory Bewsh², Shankaranarayana Bhat ${ }^{3}$, Ramesh Babu ${ }^{4}$

HOW TO CITE THIS ARTICLE:

Samith Ahmed, Gregory Bewsh, Shankaranarayana Bhat, Ramesh Babu "Low level laser therapy: healing at the speed of light". Journal of Evolution of Medical and Dental Sciences 2013; Vol2, Issue 39, September 30; Page: 7441-7463.

KEYWORDS: Laser, ATP, Mitochondria, Wound.

UNDERSTANDING THE MECHANISMS OF LOW LEVEL LASER THERAPY (LLLT): Low Level Laser Therapy (LLLT) is a rapidly growing modality used in rehabilitation and physical therapy. A number of safe and beneficial therapeutic effects of LLLT have been reported in numerous clinical conditions; however, despite many reports of positive findings from experiments conducted in-vitro, in animal models and in randomized controlled clinical trials worldwide, the use of this scientifically grounded, non-invasive, anti-inflammatory and regulatory modality has yet to find mainstream adoption by medical doctors. The aim of this review is six fold:

1) Introduce the unfamiliar reader, with some medical background, to the contemporary concept of LLLT and its pathophysiological significance,

2) Review the role of the mitochondrial pathway in the mechanisms of LLLT,

3) Provide necessary practical guidance based on personal scientific and clinical experience,

4) Assist manufacturers in their research and development,

5) Help health care practitioners choose and use an adequate light therapy device

6) Outline the prospects of LLLT as an avenue to treat chronic inflammation and pain and to aid in an effective clinical practice.

THE STATE OF THE ART: Low Level Laser Therapy (LLLT) is the drug-free, non-invasive and safe (FDA recognised) clinical application of light (usually produced by a low to mid power coherent lasers or non-coherent light emitting diodes (LEDs) in the range of $1 \mathrm{~mW}-500 \mathrm{~mW}$ ) to a patient to promote tissue regeneration, reduce inflammation and relieve pain. The light is typically of narrow spectral range (1 to $40 \mathrm{~nm}$ bandwidth) in the visible (red) 600 to $700 \mathrm{~nm}$ or near infrared (NIR) spectrum (700nm to $950 \mathrm{~nm}$ ), achieving an average power density (irradiance) between. 001 to 5 $\mathrm{W} / \mathrm{cm}^{2}$.

Light irradiation is typically applied onto the affected area (the areas where the treatment is needed) or pain projected area or suggested acupuncture points for a few seconds to several minutes, daily, or multiple times per week, for a treatment period of up to 8 to 10 weeks depending on chronicity. Thus in treatment frequency and duration LLLT does not differ from other modalities, such as thermal heating blankets ${ }^{(1)}$, electrostimulation ${ }^{(2)}$ or ultrasound $^{(3)}$; however, as we shall see dramatically different in cellular mechanism and efficacy.

LLLT is not considered an ablative or destructive procedure on the tissue or cellular level and employs biomodulation via photophysical and potential photochemical mechanisms, comparable to photosynthesis in plants; whereby, the absorbed light is able to initiate a cascade of molecular and functional regulatory changes. 
The reason why the technique is generally referred to as Low Level Laser Therapy (LLLT) is that the optimal level of energy density delivered to the tissues is low when compared to other forms of laser therapy such as the densities required for ablation, cutting, and thermally coagulating tissue. In general, the power densities used for LLLT are lower than those needed to produce heating of tissue (i. e. less than $500 \mathrm{mWcm}-2$, depending on wavelength and tissue type), and the total energy delivered to the treated area is commonly below $1 \mathrm{~J} \mathrm{~cm}^{-2}$. A common reference is the "Guidelines for Skin Exposure to Laser Light" in the International Standards Manual (IEC-825) which considers exposure below $200 \mathrm{~mW} \mathrm{cm-2}$ as a safe exposure (4) in the visible range, escalating to approximately $500 \mathrm{~mW} / \mathrm{cm}^{2}$ in the near infrared range.

The use of low levels of visible or near infrared light (LLLT) (5), for reduction of inflammation, elimination of pain (6), healing of wounds, repair to nerves injuries and (7) prevention of tissue damage by reducing cellular apoptosis (for example, due to ischemia and reperfusion injury), has been known for fifty years since shortly after the invention of lasers in the early 60s.

Despite many reports of positive findings from experiments conducted in-vitro, in-vivo (animal and human models) and in randomized controlled clinical trials, the mechanisms of action of LLLT in biological tissue remains not fully elucidated. This likely is due to two main reasons: firstly the complexity in interpretation of scientific and clinical data generated in different labs and clinical settings and secondly variability in the use of light sources (medical devices) and treatment protocols utilized including, illumination parameters (such as: wavelength, fluence, power density, pulse structure, etc.) and the treatment schedule. These factors lead to many machinations of factors that make side to side comparisons difficult.

As a result, the original field of Low Level Laser (coherent-monochromatic light) Therapy (LLLT) or Low Level Light Therapy (LLLT) subject has now expanded to include photo biomodulation and photobiostimulation using non-laser (non-coherent light) instruments.

These variation in light sources utilized have led to an increase in the number of negative studies and created some controversy despite the overwhelming number of positive clinical results $^{(1)}$. It is noteworthy that many studies have been conducted without proper scientific methodology, as all the characteristics of the light emitted by lasers or LEDs must be specified if a paper is to be useful. A requirement for a good phototherapy study, using low intensity radiation in the visible or near-infrared region, whether from a laser, an LED, or a filtered incandescent lamp is to specify everything about the light source, i. e., wavelength(s), power, dose, area of exposure, time, etc. There are numerous published experimental and clinical studies that were conducted with good scientific methodology, but unfortunately they did not describe the light source ${ }^{(8)}$; therefore, these studies cannot be repeated or extended by another author. Such publications are useless for progressing the field of science of LLLT.

In recent years, major advances have been made in understanding the mechanisms that operate at the cellular and tissue levels during LLLT. Mitochondria are thought to be the main site for the initial effects of light. The discovery and universal acceptance of this phenomenon is of particular significance to Laser manufactures, because both mitochondria membrane lipids and the large mitochondrion transmembrane protein complex (cytochrome c oxidase) have absorption spectra and action spectra peaks in the red and near infrared regions of the electromagnetic spectrum matching the emission spectra of the $660 \mathrm{~nm}$ and $905 \mathrm{~nm}$, dual wavelengths of Theralase's 
LLLT devices. Thus a photochemical activation of pathways involving these transmembrane protein complexes as well as potential subcellular photothermal effects is possible (6).

New discoveries in the last decade significantly altered our view on mitochondria. They are no longer viewed as energy-making cellular compartments but rather individual cells within- thecell. In particular, it has been suggested that many important cellular mechanisms involving specific enzymes and ion channels, such as nitric oxide synthase (NOS), ATP-dependent K+ (KATP) channels, and poly-(APD-ribose) polymerase (PARP), have a distinct, mitochondrial variant. For example, the intriguing possibility that mitochondria are significant sources of nitric oxide (NO) via a unique mitochondrial NOS variant has attracted intense interest among research groups because of the potential for NO to affect functioning of the electron transport chain (8).

It has been shown that LLLT mediated effect may employ inducible nitric oxide synthase (iNOS) to potentially activate production of nitric oxide (NO) in mitochondria (6). The discovery of this phenomenon supports a concept that NO and its derivatives (reactive nitrogen species) have multiple effects on mitochondria that may impact a cell's physiology and the cell's cycle (7).

It was shown that inducible in mitochondria nitric oxide (iNO) inhibits mitochondrial respiration via: (A) an acute and reversible inhibition of cytochrome c oxidase by NO in competition with 02 , and (B) irreversible inhibition of multiple sites by reactive nitrogen species. iNO stimulates reactive oxygen and nitrogen species production from mitochondria via respiratory inhibition, reaction with ubiquinol and reaction with 02 in the mitochondrial membranes. (9) Oxidants/free radicals may also confer physiological functions and cellular signaling processes (10) due to iNO.

According to Brown et al., mitochondria may produce and consume NO and NO stimulates mitochondrial biogenesis, apparently via upregulation of nucleotides like ATP and transcriptional factors like nuclear factor kappa B (NF kb) (9).

Therefore, it can be suggested that the super pulsed 905nm LLLT-induced NO can reprogram cellular function, mainly via oxidative stress and changes of mitochondrial temperature gradient due to process known as selective photo thermolysis and consequently initiate a cascade of local and systemic therapeutic signaling (6).

These signal transduction pathways in turn may lead to increased cell activation and traffic, modulation of regulatory cytokines, growth factors and inflammatory mediators, and expression of protective (anti-apoptotic) proteins $(11 ; 12)$.

The results of these molecular and cellular changes in animals and patients integrate such benefits as increased: healing in chronic wounds, improvements in sports injuries and carpal tunnel syndrome, pain reduction in arthritis and neuropathies, amelioration of damage after heart attacks, stroke, nerve injury and alleviation of chronic inflammation and toxicity (13).

The LLLT-induced NO may explain 1) generation of reactive oxygen species (ROS), induction of transcription factors and increased ATP production (in cells) 2) modulation of immune responses, reduction and prevention of apoptosis, regulation of circulation and angiogenesis (in tissues), and suppression of local and systemic inflammation and pain. LLLT-induced ROS, iNO and ATP mediated signaling cascades are well documented in the peer-reviewed literature $(14 ; 15 ; 16 ; 17 ; 18)$.

More evidence now suggests that LLLT is a rapidly growing modality used in physical therapy, chiropractic and sport medicine and increasingly entering mainstream medicine. A full spectrum of potential clinical targets that can be successfully treated by LLLT is continuing to grow and at this point is not exhausted. LLLT is used to increase wound healing and tissue regeneration, 
to relieve pain and inflammation, to prevent tissue death and to mitigate degeneration in many neurological indications. We believe that further advances in elucidation of the LLLT's mechanisms will lead to greater acceptance of LLLT as the therapy of choice in the established market segments and additionally as a first line or adjuvant therapy in other serious medical applications, currently not being considered typical indications.

A SHORT HISTORY OF LLLT: Light therapy is one of the oldest therapeutic methods used by humans; (historically as solar therapy by the Egyptians, Aztecs, Greeks and Romans and later as nonionizing UVB phototherapy of lupus vulgaris for which Niels Ryberg Finsen won the Nobel Prize for Physiology in $1903(14 ; 15)$.

The majority of the authors agreed that the era of LLLT starts in 1967, a few years after Gordon Gould coined the acronym LASER (Light Amplification by Stimulated Emission of Radiation) and described the essential elements for constructing a laser (1957) and 3 years after the Nobel Prize for Physics (1964) was given to Nikolay Gennadiyevich Basov, Aleksandr Mikhailovich Prokhorov and Charles Hard Townes for the invention of the MASER (microwave amplification by stimulated emission of radiation) and the laser (16). The year 1967 is important in the history of LLLT because of the ground breaking publication by Endre Mester and his colleagues (from Semmelweis University, Budapest, Hungary). The authors uniquely demonstrated the therapeutic benefit of monochromatic visible light and commenced the therapeutic laser field of science.

The investigators original expectation was to test if laser radiation might cause cancer in mice. Mice were shaved and divided into control and treatment groups. The treatment group received the light treatment with a low powered ruby laser $(694 \mathrm{~nm})$ while the control group did not. The treated animals did not develop any malignancies and the treatment was safe; however, the authors made a surprising observation about more rapid dorsal hair regrowth in the treated group than the untreated group (17). Remarkably, the same study data can be considered as the first disclosure of potential safety and efficacy of LLLT, particularly attributed to LLLT's biostimulation abilities.

Since then, medical treatment with coherent light sources (lasers) or non coherent light (LEDs) has passed through its experimental stage and is firmly rooted in clinical and scientific documentation. Currently, low level laser (or laser therapy LLLT), also known as "cold laser", "soft laser", "biostimulation" or "photo biomodulation" is recognised by the FDA and practiced as part of physical therapy in many parts of the world (18).

LLLT is showing promise in the treatment of a wide variety of medical conditions and has been proven to be clinically safe and beneficial therapeutically in many tissues and organs (Figure 1). 


\section{REVIEW ARTICLE}

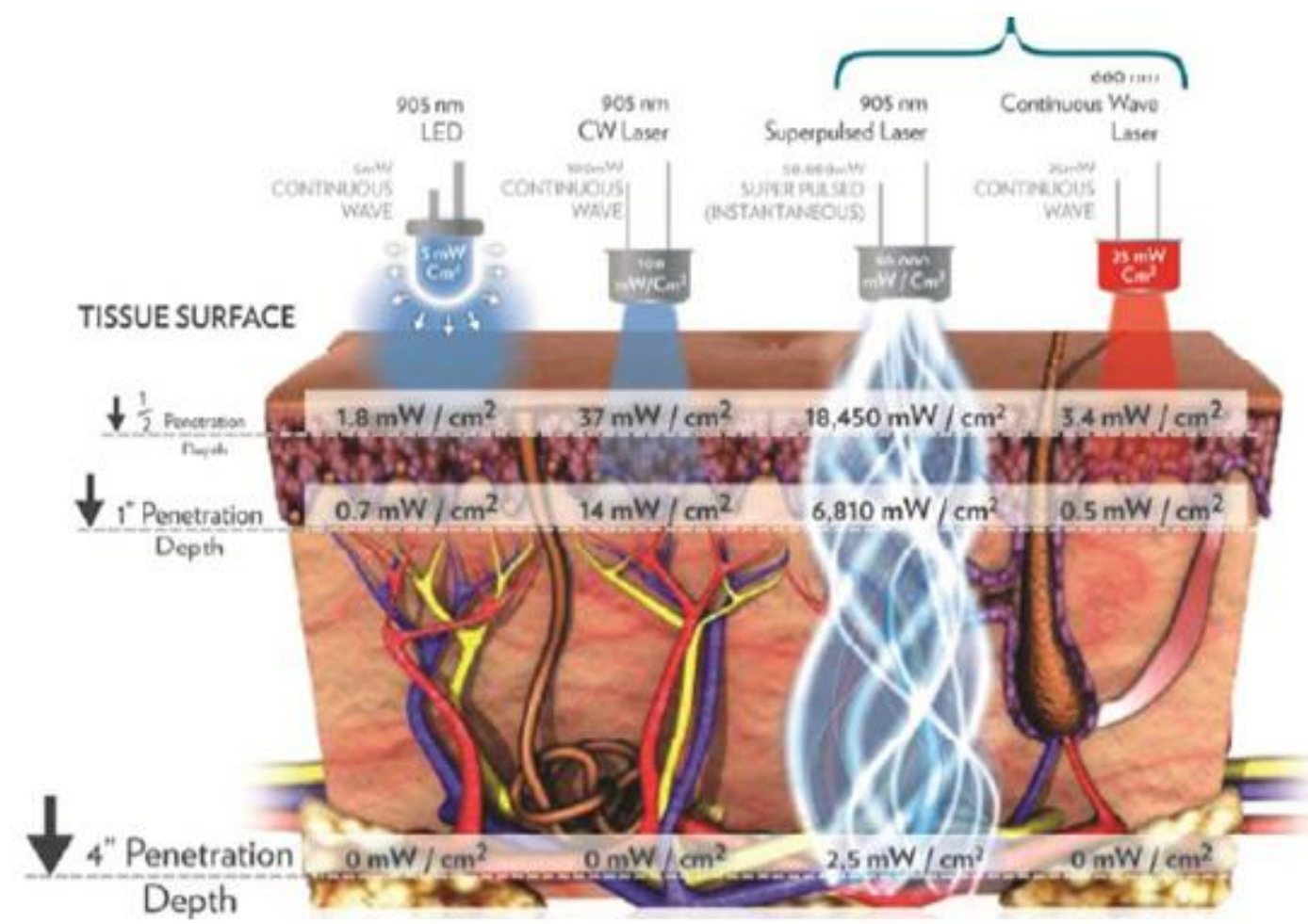

Figure 1: The diagram below represents the difference in depth of penetration between therapeutic lasers.

Currently, the knowledge of LLLT mechanisms continues to expand. Physicians are now aware of LLLT's potential to induce cellular and tissue effects through; for example, accelerated ATP production and molecular signaling and that LLLT can be very effective in the treatment of various serious clinical disorders.

In clinical use, however, it is often difficult to predict patient response to LLLT. It appears that several key features, such as: the LLLT parameters and the treatment regimen (including, irradiance [mW cm-2], radiant exposure [J cm-2], treatment regime (including the treatment mode, pulsed vs. CW, and the treatment schedule), light attenuation [cm-2] in the tissues, etc.), cellular pathophysiological status (including, reduction=oxidation (redox state), a disease localisation (depth under the surface $[\mathrm{mm}]$ ) tissue characteristics (including the tissue scattering parameters), the character and the level of inflammatory process and variability of physiological and clinical conditions in patients, can play a central role in determining sensitivity (and hence clinical efficacy) to LLLT and may help to explain intra-individual variability in patient responsiveness to the conducted therapy. Various cellular responses to visible and infrared radiation have been studied for decades in the context of molecular mechanisms of low level non-coherent (non-laser) light and laser phototherapy $(19 ; 20 ; 21 ; 22)$.

It is generally accepted that the mitochondria are the initial site of light action in mammalian cells, and cytochrome c oxidase (the terminal enzyme of the mitochondrial respiratory chain) is one of the key responsible molecules (23) particular due to its broad absorption in the visible red and NIR spectrum. 
THE KEY MITOCHONDRIAL MECHANISMS OF LLLT: It is believed that in mammalian organisms the mitochondrial changes taking place are playing the essential role in the mechanisms of LLLT and, according to Hamblin and colleagues (2010), the response of cells to light is determined by the mitochondrial number, their activity and membrane potential (24).

Mitochondria are the energy-transducing organelles of eukaryotic cells in which fuels that drive cellular metabolism (i. e., carbohydrates and fats) are converted into adenosine triphosphate (ATP) through the electron transport chain and the oxidative phosphorylation system (the "respiratory chain", Figure 2(29; 30).

Mitochondria are also involved in calcium buffering and the regulation of apoptosis (25). They arose as intracellular symbiont in the evolutionary past, and can be traced to the prokaryote $\alpha$ proteobacterium (26). There are hundreds to thousands of mitochondria per cell (27), somewhat dependent on the energy requirement of the individual cell. Structurally, mitochondria have four compartments: the outer membrane, the inner membrane, the intermembrane space, and the matrix (the region inside the inner membrane, see Figure $2,(30 ; 33 ; 34)$.

The respiratory chain is located on the inner mitochondrial membrane. It consists of five multimeric protein complexes: reduced nicotinamide adenine dinucleotide (NADH) dehydrogenaseubiquinone oxidoreductase (complex I), succinate dehydrogenase ubiquinone oxidoreductase (complex II), ubiquinone-cytochrome c oxidoreductase (complex III), cytochrome c oxidase (complex IV), and ATP synthase (complex V). In addition, the respiratory chain requires 2 small electron carriers, ubiquinone and cytochrome c.

Energy generation via ATP synthesis involves two coordinated processes: 1) electrons (hydrogen ions derived from NADH and reduced flavin adenine dinucleotide) are transported along the complexes to molecular oxygen, resulting in the production of water; and 2) simultaneously, protons (hydrogen ions) are pumped across the mitochondrial inner membrane (from the matrix to the intermembrane space) by complexes I, III, and IV. ATP is generated by the influx of these protons back into the mitochondrial matrix through complex V (ATP synthase (27)).

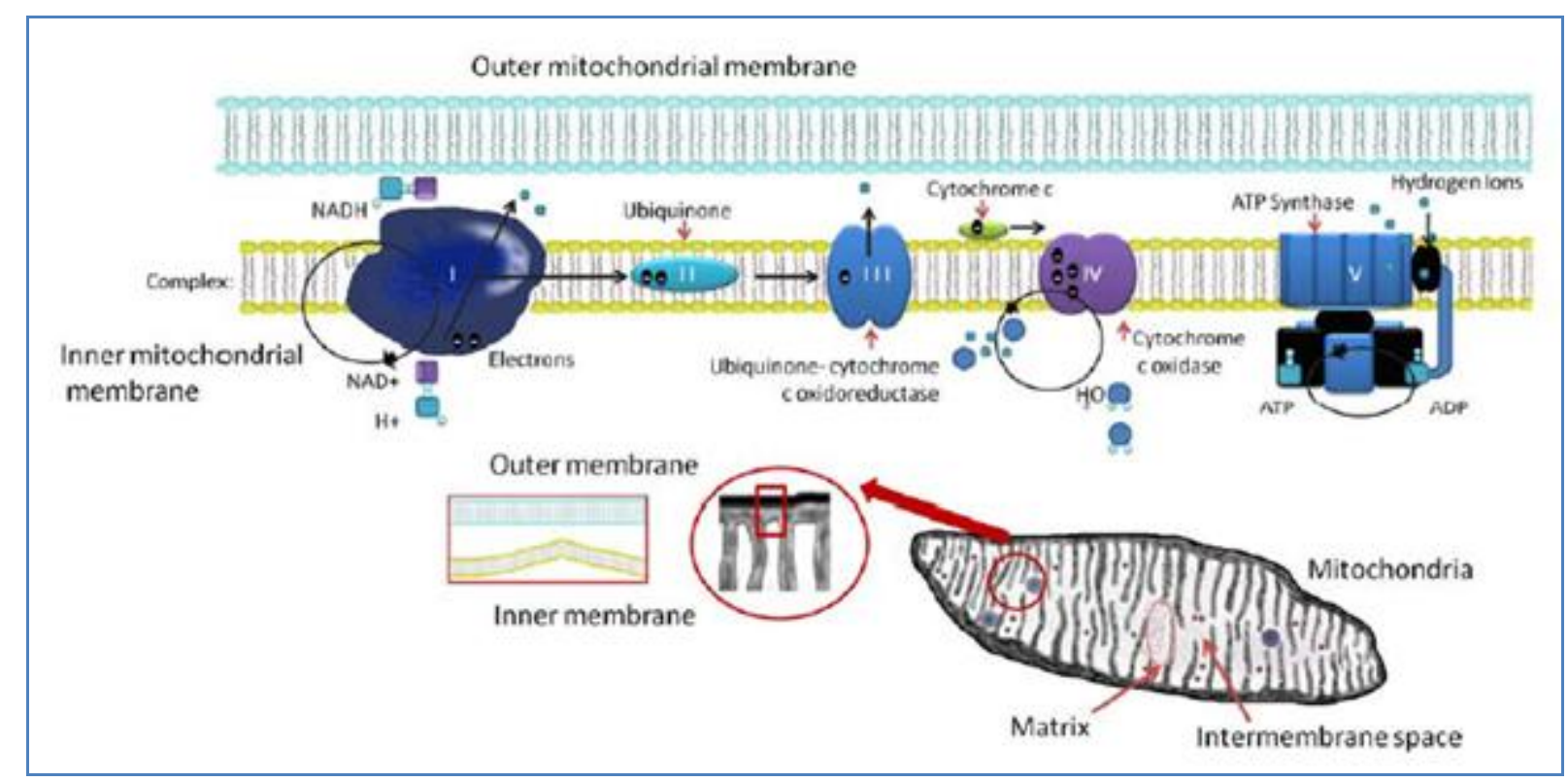

Figure 2: Mitochondria and Mitochondrial respiratory chain.

Journal of Evolution of Medical and Dental Sciences/ Volume 2/ Issue 39/ September 30, 2013 
Karu et al. (2004) proposed that biological effects of visible and near-IR light, in mammalian cells, are initiated via the mitochondrial signaling pathway ${ }^{(35)}$. The authors also suggested that the redox absorbance recorded in the spectral range close to 600-900 $\mathrm{nm}$ changes in living cells (28).

These observations are of particular interest because a growing number of recent scientific reports and observations are providing more support to the concept that the functions of mitochondria go beyond the generation of ATP and the regulation of energy metabolism (29).

It has been shown that mitochondria are playing a major role as an integrator of intrinsic and extrinsic cellular signals, which can affect the health and survival of the cell; as well as, may play an essential role in the cell-to-cell communication signaling mechanisms (30). ATP binding to the extracellular surface of $\mathrm{P} 2 \mathrm{Y}$-purinergic receptors, allows release of cytosolic Ca2+calcium, initiates regulatory gene expression and induces a cascade of intracellular molecular signaling (31).

Both $\mathrm{Ca} 2+$ uptake and efflux from mitochondria consume the mitochondrial membrane potential $(\Delta \Psi \mathrm{mt})$; and, in this way modify the mitochondrial activity (and therefore the ATP synthesis), which can be regulated by LLLT.

From a clinician's point of view (for selection of the most effective parameters and appropriate LLLT's medical devices) this scientific data provides a new practical guideline and assistance towards a better understanding of the biomolecular mechanisms of LLLT. Recent reports have demonstrated that ATP is a critical signaling molecule that allows cells and tissues throughout the body to communicate with one another (32).

This new aspect of ATP as an intercellular signaling molecule allows broadening of the understanding of the cellular and molecular mechanisms of LLLT, and hence provides grounds for optimization of LLLT efficacy. It has been shown that neurons and other cells may release ATP into muscle, gut, and bladder tissue as a messenger molecule. The specific receptors (a family of P2, purinergic receptors) for ATP signaling were found and identified (31; 32).

ATP activation of $\mathrm{P} 2$ receptors (subtypes $\mathrm{P} 2 \mathrm{X}$ and $\mathrm{P} 2 \mathrm{Y}$ ) can produce various biological effects. A recent publication by Anders et al. (2008), demonstrated that P2Y2 and P2Y11 receptors were expressed in near-IR light irradiated normal human neural progenitor cells in vitro (33). These observations support the notion that the irradiation of cells with near-IR light in the interval close to $905 \mathrm{~nm}$ could be functioning as a surrogate for growth factors. Recent reviews indicate that laboratories worldwide are now racing to turn the data about ATP as a neurotransmitter into therapies (31).

As a neurotransmitter, ATP is directly involved in brain function, sensory reception, and the neuron system control of muscles and organs. When released by neuronal and non neuronal cells, it often triggers protective responses, such as bone building and cell proliferation $(17 ; 39)$. The LLLTinduced ATP's signaling mechanism also is believed to be involved in its use during pain therapy (29); it is needless to say that chronic and neuropathic pains are the first disorders treated successfully with LLLT many decades ago.

The role of ATP as a signaling molecule provides a new basis for explaining the versatility of LLLT effects (34). Numerous reports indicate that light could regulate gene expression via mitochondrial mechanisms. For example, Hu et al., (2007) have shown that mitochondria play a central role in cellular homeostasis, and their homeostatic center is the mitochondrial membrane potential (mt). The assessment of the mt in cells conducted by the authors indicated that A2058 cells 
treated with dosages higher than $0.5 \mathrm{~J} \mathrm{~cm}-2$ He-Ne laser irradiation at $633 \mathrm{~nm}$ exhibited a marked increase in mt. They also found upregulation of cytochrome c oxidase activity, increased phosphorylation of Jun N-terminal kinase (JNK) and later, activated activator protein-1 (AP-1), which led to increased cell proliferation (35).

Mitochondrial membrane potential may play a role in response of cells to light therapy. Huang et al. (2004) used tetramethyl rhodamine methyl ester to compare the mitochondria of six different cell types. They found that the mean mt differed significantly between cell types, but that the cell area or size also differed, and that a more accurate comparison was to calculate the integrated mt over the cell cross sectional area. Although fibroblasts had a high measured value of mean $\mathrm{mt}$, when integrated over the whole cell the value was actually the lowest of the cell types tested because fibroblasts had a much greater surface area (36).

Neuronal cells had higher mean mt values in the cell bodies compared to the growth cones. Further work is necessary to determine if mean $\mathrm{mt}$ or mean mt per cell area correlated with measures of cellular response to light. However, there is more supportive evidence that cell types with greater overall mitochondrial activity (for instance cortical neurons and cardiomyocytes) do in fact respond well to light. The presented observations may suggest that cells with a high level of mitochondrial activity (or high $\mathrm{mt}$ ) may have a higher response to light than cells with low mitochondrial activity (37).

The majority of authors agree that mitochondria are the key cellular targets, able to initiate LLLT induced biologic events, leading to: upregulation of ATP production, induction of various transcription factors and modulation of pro and anti-inflammatory gene expression. These effects in turn may lead to cell biostimulation, modulation of the levels of various inflammatory mediators (such as cytokines), release of the growth factors, as well as, increase in tissue oxygenation and reparation. The results of these biological interactions in animals and humans include such therapeutic effects as: acceleration in healing of chronic wounds, improvements in sport injuries, reduction of inflammation and pain in arthritis and neuropathies, amelioration of toxicity and tissue damage after infectious diseases and heart attacks, and restoration of vascular and neuronal damage (38).

THE ROLE OF THE 905 nm NIR COHERENT (LASER) LIGHT IN LLLT: An alternative explanation for LLLT effects (particularly in the near-IR wavelength region) on cells and mitochondria does not rely on specific photon absorption by proteins and/or enzymes and their defined absorption bands leading to photochemistry, but relies more on specific photon absorption leading to localized photothermal effects, confined to sensitive structures, such as lipids through non-specific photon absorption. The quantum energy of photons at $905 \mathrm{~nm}$ is only $\sim 1.3 \mathrm{eV}$ and hence insufficient to achieve an electronic excitation in most biomolecules, including cytochrome c oxidase, making a vibrational or thermal activation of these target molecules more likely at this wavelength.

Since the total energy per photon delivered is small the resulting rise in average tissue temperature would be insignificant if this energy was evenly distributed over the whole cell. However in the case of coherent laser light the energy is not evenly distributed, but forms a speckle pattern at any given instance in time. When a surface is illuminated by a light wave, according to diffraction theory, each point on an illuminated surface acts as a source of secondary spherical waves. Laser speckle is formed by interference (either constructive or destructive) of waves that 
have been scattered from each point on the illuminated surface. If the surface is rough enough to create path length differences exceeding one wavelength, giving rise to phase changes greater than 2 , the amplitude, and hence the intensity, of the resultant light varies randomly, from 0 to twice the average intensity. As tissue is not static these speckle pattern are varying over time and after less than $1 / 100$ th of a second the temporal average of the intensity is again established across the tissue. If light of low coherence (i. e. made up of many wavelengths moving out of phase) is used, a speckle pattern will not normally be observed, because the speckle patterns produced by individual wavelengths have different dimensions and will normally cancel one another out. The "size" of the speckles is a function of the wavelength of the light, the surface area of the laser beam that illuminates the first surface, and the distance between this surface and the plane where the speckle pattern is formed. In tissue, the diameter of the speckles is of the order of the wavelength of light i. e. about 1 micron and thus comparable to the size of the mitochondria. While continuous wave light sources speckle pattern are averaged temporally as stated above they do not do so if the light pulse is shorter than the speckle relaxation time. Thus the hypothesis is that laser speckles produce by super pulsed laser sources can produce micro-thermal gradients due to inhomogeneous energy absorption that can stimulate or otherwise alter the metabolism within mitochondria (39).

This hypothesis would explain reports that some LLLT effects in cells and tissues are more pronounced when coherent pulsed laser light is used than comparable non-coherent light from LED or filtered lamp sources that is of similar wavelength range although not monochromatic (40). For these micro-thermal gradients to be effective the pulse repetition rate needs to be slow enough to allow a complete relaxation of these gradients (for micron sized 'hot spots' this relaxation time is in the millisecond range) prior to the generation of a novel speckle pattern generated micro-thermal pattern.

Lilge, et al., (2009) used near-IR light at $905 \mathrm{~nm}$ (Theralase Inc.) and a high peak power of $50 \mathrm{~W} \mathrm{~cm}-2$, suggested a role of localized thermal activation (or selective photothermolysis) within mitochondria in the mechanisms of super pulsed $905 \mathrm{~nm}$ LLLT $(5 ; 48 ; 49)$.

The authors suggest that selective photo thermolysis occurs if photon absorption within a target structure is much higher than in the surrounding tissue, but the pulse duration is short compared to the thermal relaxation time of the target structure. Two hundred nanosecond pulses of $905 \mathrm{~nm}$ laser light, used by the investigators, exceed the thermal relaxation time of structures smaller than $200 \mathrm{~nm}$. Hence, according to the authors, the cell membrane ( $20 \mathrm{~nm}$ ) would radiate too much energy to the surrounding area not the thermal energy and thus not achieving elevated temperatures, hence presenting an unlikely target structure.

Huttmann and colleagues (1999) suggested that the inverse of the repetition rate of $1 / 10$ $\mathrm{kHz}$ or 100 microseconds needs to be equivalent to at least several thermal relaxation times of the target structure, permitting their cooling between pulses to avoid accumulation of heat, which would finally exceed the Arrhenius damage integral (41). Therefore, the target structure would be required to be smaller than 3 to $5 \mu \mathrm{m}$, but larger than $200 \mathrm{~nm}$.

Mitochondria membranes have high amount of lipids with peak absorbance at 900 to $930 \mathrm{~nm}$, close to the super pulsed $905 \mathrm{~nm}$ wavelength used. Their inherent size (a hydrophilic head group <, 1. $0 \mathrm{~nm}$; and a hydrophobic core of the bilayer is typically 3. $0-4.0 \mathrm{~nm}$ thick,) and the possibility for lipids to be the main absorber make mitochondria lipid structures the most apparent targets for 
near-IR-induced selective photo thermolysis (6). Note these micro-thermal gradients and selective photo thermolysis effects exist only for super pulsed light sources.

LLLT exhibits diverse effects on pathophysiologic processes according to varying wavelengths and wave modes. It is could be suggested that the LLLT biomechanisms induced by 905 $\mathrm{nm}$ near infrared light, particularly upregulation by the nitric oxide pathway following the $905 \mathrm{~nm}$ wave in the super pulsed mode, are not the same when compared to visible light.

Several additional scientific studies suggest that mitochondria are accountable for the cellular response to LLLT. Passarella et al., (1984) reported that LLLT affect both transport and enzymatic processes in cells by acting on the cell's mitochondria and increasing the ATP synthesis via oxidative phosphorylation (42).

Taken together, this clinical and scientific data strongly indicate the essential role of near- IR laser light in the biomechanisms of LLLT healing. It can also be concluded that local transient heating of particular cellular micro regions with a size characteristic of mitochondria based chromophores is possible and this effect may contribute to the efficacy of LLLT. The local transient rise in temperature may cause structural (conformational) changes of near-IR light absorbing molecules and trigger secondary biochemical and other biomolecular activity such as activation or inhibition of enzymes and gene expression. The opportunity of local transient heating of absorbing chromophores, which is offered by the $905 \mathrm{~nm}$ super pulsed technology, takes medical applications of modern LLLT to entirely new levels and clinical significance when whole anatomical tissues are irradiated during patient treatment.

THE ROLE OF THE $660 \mathrm{~nm}$ COHERENT (LASER) RED LIGHT IN LLLT: In addition to the nearinfrared light at super pulsed $905 \mathrm{~nm}$ wavelengths, the most highly LLLT technologies encompasses biomechanisms mediated by coherent visible red light at $660 \mathrm{~nm}$ wavelength or approximately 1. 9 eV per photon.

The most recent, well defined, in-vivo data suggests the presence of biological mechanisms mediated by $660 \mathrm{~nm}$ laser light. Studying the effect of LLLT on inflammatory pain, the authors have shown that Low-Level Laser phototherapy [InGaAIP visible laser diode (660 nm) with a radiant exposure of $2.5 \mathrm{~J} \mathrm{~cm}-2$ ] was proven to be an effective analgesic, while non-coherent LED light did not show a similar effect (22). Similar to the $905 \mathrm{~nm}$ speckle pattern exists a comparable time albeit at smaller dimensions for $660 \mathrm{~nm}$ red laser light. Increased RNA and protein synthesis was demonstrated after HeNe laser $(632.8 \mathrm{~nm})$ treatment at $5 \mathrm{~J} \mathrm{~cm}-2$ (43).

In 1994, Pastore et al (2004), found increased activity of cytochrome c oxidase and an increase in polarographically measured oxygen uptake after $2 \mathrm{~J} \mathrm{~cm}-2$ of HeNe $(633 \mathrm{~nm})$ irradiation. As a major stimulation in the proton pumping activity, an increase of about $55 \%$ in the $\mathrm{H}+$ /e- ratio was found in illuminated mitochondria indicating a role of the mitochondrial changes in the mechanisms of red laser light based therapy (44).

W. Yu et al., 1997, used $660 \mathrm{~nm}$ laser at a power density of $10 \mathrm{~mW} \mathrm{cm-1}$ and showed increased oxygen consumption following $(0.6 \mathrm{~J} \mathrm{~cm}-2$ and $1.2 \mathrm{~J} \mathrm{~cm}-2)$ radiance exposure, increased phosphate potential, and energy charge (at $1.8 \mathrm{~J} \mathrm{~cm}-2$ and $2.4 \mathrm{~J} \mathrm{~cm}-2$ ) and enhanced activities of NADH: ubiquinone oxidoreductase, ubiquinol: ferri cytochrome c oxidoreductase and ferrocytochrome c : oxygen oxidoreductase (between $0.6 \mathrm{~J} \mathrm{~cm}-2$, and $4.8 \mathrm{~J} \mathrm{~cm}-2$ ) (45). 
Originally, Gordon and Surrey (1960) found that cells exposure to light of $660 \mathrm{~nm}$ showed enhanced ATP synthesis and may alter the phosphorylative capacity in mitochondria upon direct exposure of the organelles in this red wavelength diapason. $(46 ; 47)$.

In 2005, these original observations were confirmed by Amat et al., (48), and very recently (2011) by Alghamdi and colleagues (49).

Yu et al., (1997) studied whether oxidative metabolism and electron chain enzymes in liver mitochondria can be modulated by $660 \mathrm{~nm}$ wavelength photo irradiation. The oxygen consumption, phosphate potential, and energy charge of liver mitochondria were determined following photo irradiation (45).

It is generally believed that mitochondria are the primary affected organelle in mammalian tissue irradiated with laser light at $660 \mathrm{~nm}$ and/or $905 \mathrm{~nm}(59 ; 60)$ albeit, possibly different mechanisms exist ranging from direct photochemical to photothermal initiated effects.

This assumption led to the development of the highest efficacy advanced LLLT systems that, as described in detail below, encompass potent and complementary bioregulatory mechanisms of $660 \mathrm{~nm}$ (red) laser light and $905 \mathrm{~nm}$ (near infrared) super pulsed laser light at a particular therapeutic mode of administration.

There is certainly more than one reaction involved in the primary mechanisms of LLLT. There are no grounds to believe that only one of these processes occurs when a tissue is irradiated. An important question for the future research is which one of these reactions is responsible for a certain wavelength effect. However, experimental data clearly support use of $660 \mathrm{~nm}$ visible red laser light and $905 \mathrm{~nm}$ super pulsed NIR laser light based on their role in modulation of redox mitochondrial function, changes in properties of terminal enzymes and cellular signaling that might be critical steps in bioregulatory mechanisms of LLLT.

THE OPTICAL AND THERAPEUTIC WINDOWS OF LLLT: It should be noted that both absorption coefficients ( $\mu \mathrm{a}$ ) and scattering (us) coefficients of light in tissue are wavelength dependent in any given tissue. The light scattering is much more pronounced in the blue region of the visible spectrum than in the red and near infrared spectrum (50).

The data suggests that, particularly in living tissues, red light penetrates deeper than blue light, and the penetration of infrared light is deeper than red light. In addition, water in tissues begins to absorb significantly at wavelengths greater than $960 \mathrm{~nm}$, whereas wavelengths below $600 \mathrm{~nm}$ are strongly absorbed by the hemoglobins (oxy HB, and deoxy HB) in the blood.

Therefore, although light at violet blue, green, yellow, and orange, wavelengths may have various effects on cells growing in optically transparent culture medium, the use of LLLT in animals and patients almost exclusively involves light in the range above $600 \mathrm{~nm}$ and below $950 \mathrm{~nm}$ with the maximum effective "optical window" ranging from $650 \mathrm{~nm}$ to $930 \mathrm{~nm}$ (Figure 3). 


\section{REVIEW ARTICLE}

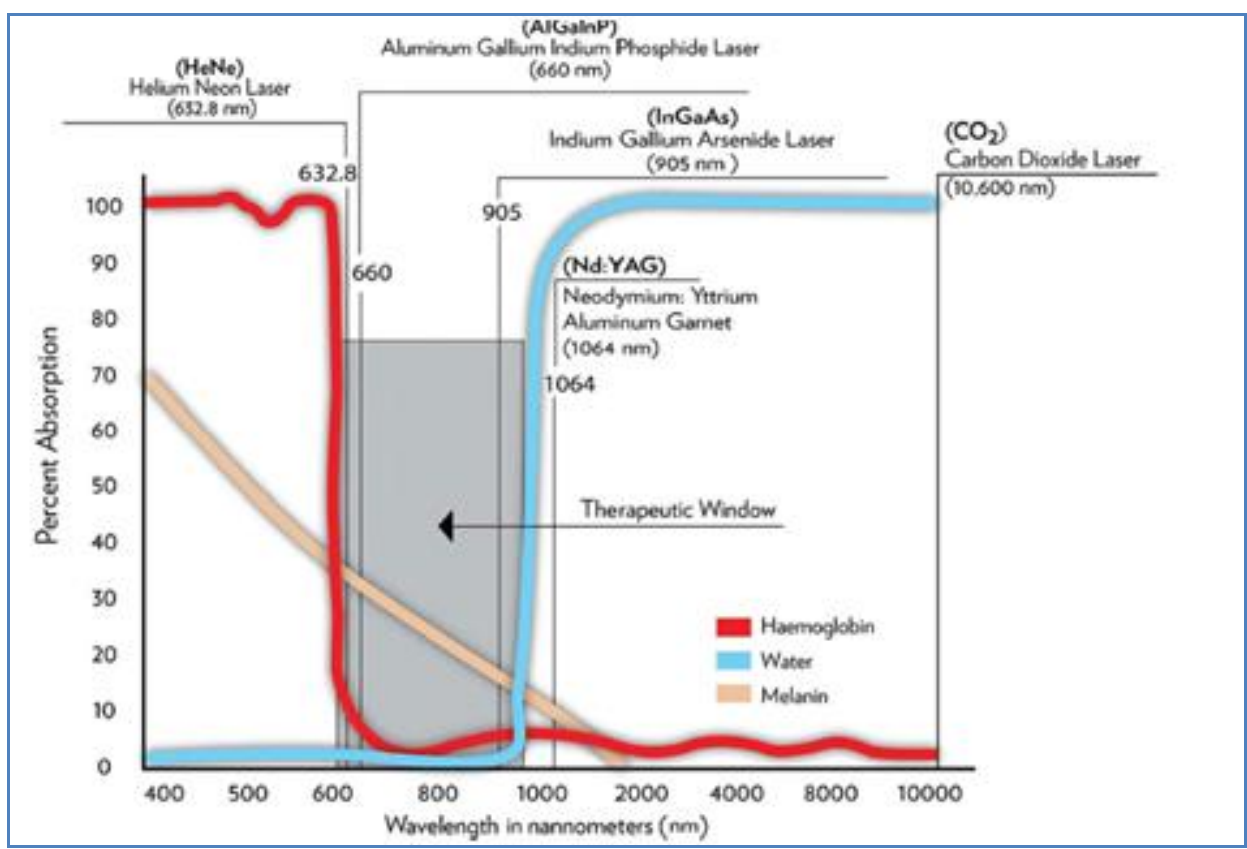

Within the optical window of light transmission it is important to consider also the change in quantum energy per photon that is dropping from $\sim 1.8 \mathrm{eV}$ at $660 \mathrm{~nm}$ to $\sim 1.2 \mathrm{eV}$ at $950 \mathrm{~nm}$ respectively. While quantum energy above $1.5 \mathrm{eV}$ can result in electronic activation of biomolecules and thus initiate direct photochemical events; this is not the case at lower quantum energies, and activation energies below $1.5 \mathrm{eV}$ cannot result in direct photochemistry. Therefore, the change in quantum energy per photon is a very important characteristic that may augment the LLLT clinical efficacy $(51 ; 52)$.

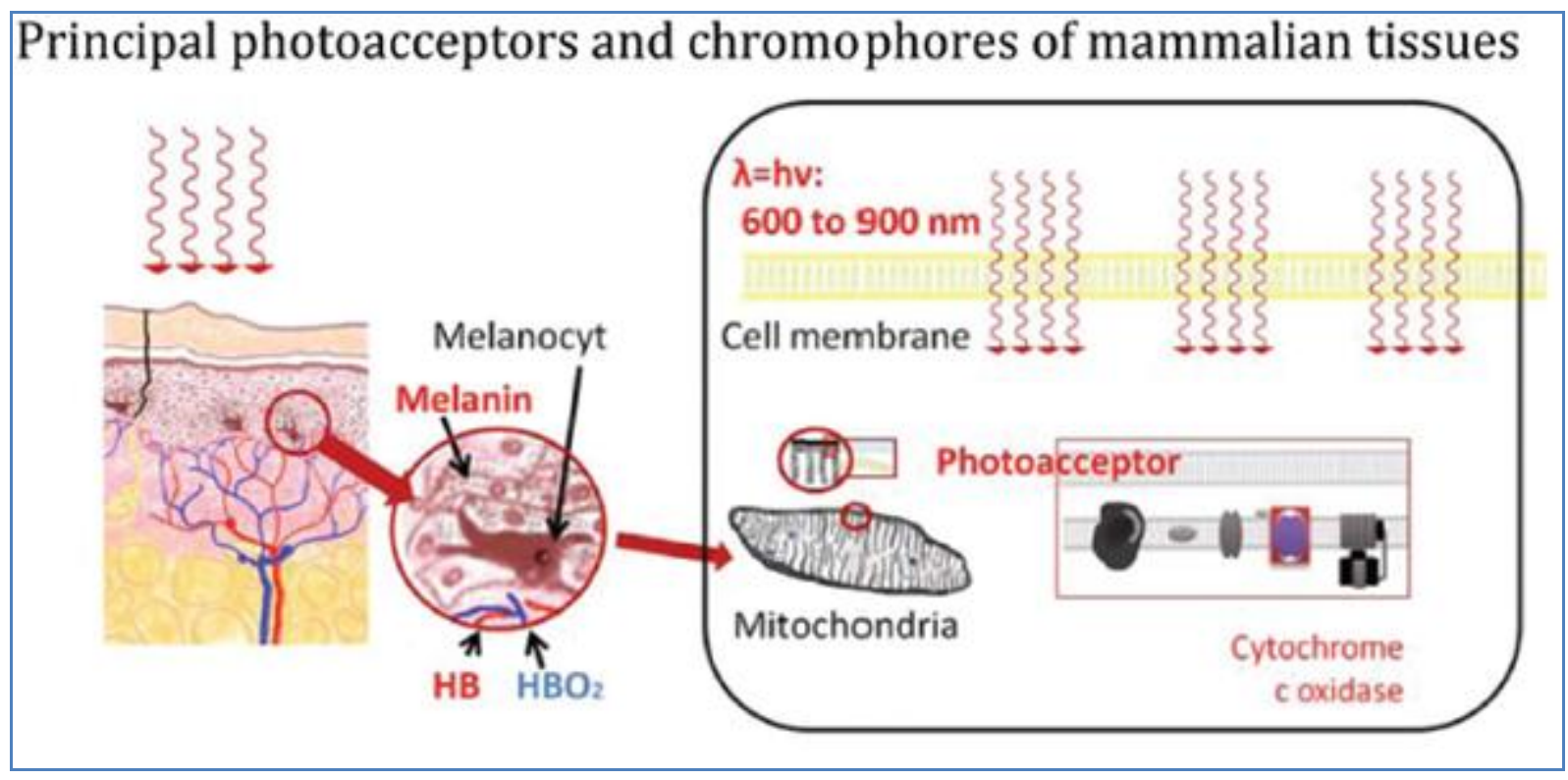

Hence, the medical device parameters are interrelated according where power densities necessary for stimulation I stim have to surpass the threshold irradiance I $(53 ; 54)$. 
According to Sommer et al., (2001) (54) Light power densities lower than the threshold values I obviously do not produce therapeutic effects, even under a prolongation of the irradiation time. The effective range is given by the particular Arndt-Schultz curve similar to that suggested by Sommer and colleagues (54). Although equation 3 is physically surprisingly simple, the biological implications are by no means trivial. Biologically, the parameters and I stim are clearly independent from each other, an important consideration at least for the medical applications of photobiological effects with the use of lasers (including incoherent light sources) at low energy density levels (54).

BIPHASIC DOSE RESPONSE IN LOW LEVEL LIGHT THERAPY: One of the key factors that affect the efficacy of LLLT is a biphasic dose response that directly correlates with the characteristics of the wavelength and the amount of energy absorbed by a chromophore or a photoacceptor which of the function of the light energy density (the radiant exposure or light fluence measured below the surface).

The important role of a biphasic response has been demonstrated by a number of studies in cell culture (55), animal models (21) and in clinical studies (56).

It has generally been observed that an optimal light irradiance and radiance exposure exists for any particular application, and irradiances lower than this optimum value, or more significantly, larger than the optimum value will have a diminished therapeutic outcome. Therefore for high doses of light (a common disadvantage of Class IV Lasers used as a therapeutic means that are able to produce an excessive amount of heat and /or generate an overwhelming level of power density deposited into the tissue) a negative outcome may even result (56), known as bioinhibition.

In connection with the versatility of LLLT effects, and because of the probable existence of a non-linear, biphasic power density response, as mentioned above, choosing the correct LLLT medical device (in terms of irradiation parameters, such as: wavelength, irradiance, pulse structure, coherence, polarization and dose characteristics) for any specific medical condition is mandatory to achieve clinically efficacious results.

In addition, there has been some confusion in the literature about the delivered fluence when the light spot is small. For example, according to Hamblin and colleagues, if 5 Joules of light is given to a spot of $5 \mathrm{~mm} 2$ the fluence is $100 \mathrm{Jcm}-2$ which is nominally the same fluence as $100 \mathrm{Jcm}-2$ delivered to $10 \mathrm{~cm} 2$, but the total energy delivered in the latter case is 200 times greater, due to the requisite increase in surface area.

Overall the concept of power density loses meaning rapidly when approaching small irradiation spot sizes. In general, the light distribution in tissue is equal for any spot size diameter smaller than $0.5 \mathrm{~mm}$, the inverse of the average reduced scattering coefficient $\mu$ 's reported for tissues in the visible and near infra-red spectral range (57).

For light wavelengths greater than $650 \mathrm{~nm}$, in most tissues and particularly in skin beam surface area should be considered when the light spot approaches a diameter of $\sim 2-3 \mathrm{~mm}$ as the angular spread of the emitted light from the diodes could double this beam surface area prior to reaching the tissue surface. In particular when laser diodes are clustered close together their beam surface areas overlap combining the individual fluence rate profiles into a single profile within the first few mm of depth into the tissue; hence, determining the effective area a can be more complex than first imagined. 
Evidence suggests that both energy density (radiant exposure) and power density (irradiance) are key biological parameters for the effectiveness of laser therapy, and they may both operate within specific thresholds (i. e., : a lower and an upper threshold for both parameters between which LLLT is effective, and outside of which LLLT is too weak [that is one of the common therapeutic disadvantages for the light sources with power levels of less than $5 \mathrm{~mW}$ (i. e., many Class I-II and IIIa light devices)] to have any effect or that intense energy densities is one of the common therapeutic disadvantages for the light sources with energy density levels greater than $10 \mathrm{Jcm}-2$ (i. e., : Class IV light devices) that the therapeutic effect is inhibited or can be in fact adverse to the subject. (see figure 5) (54).

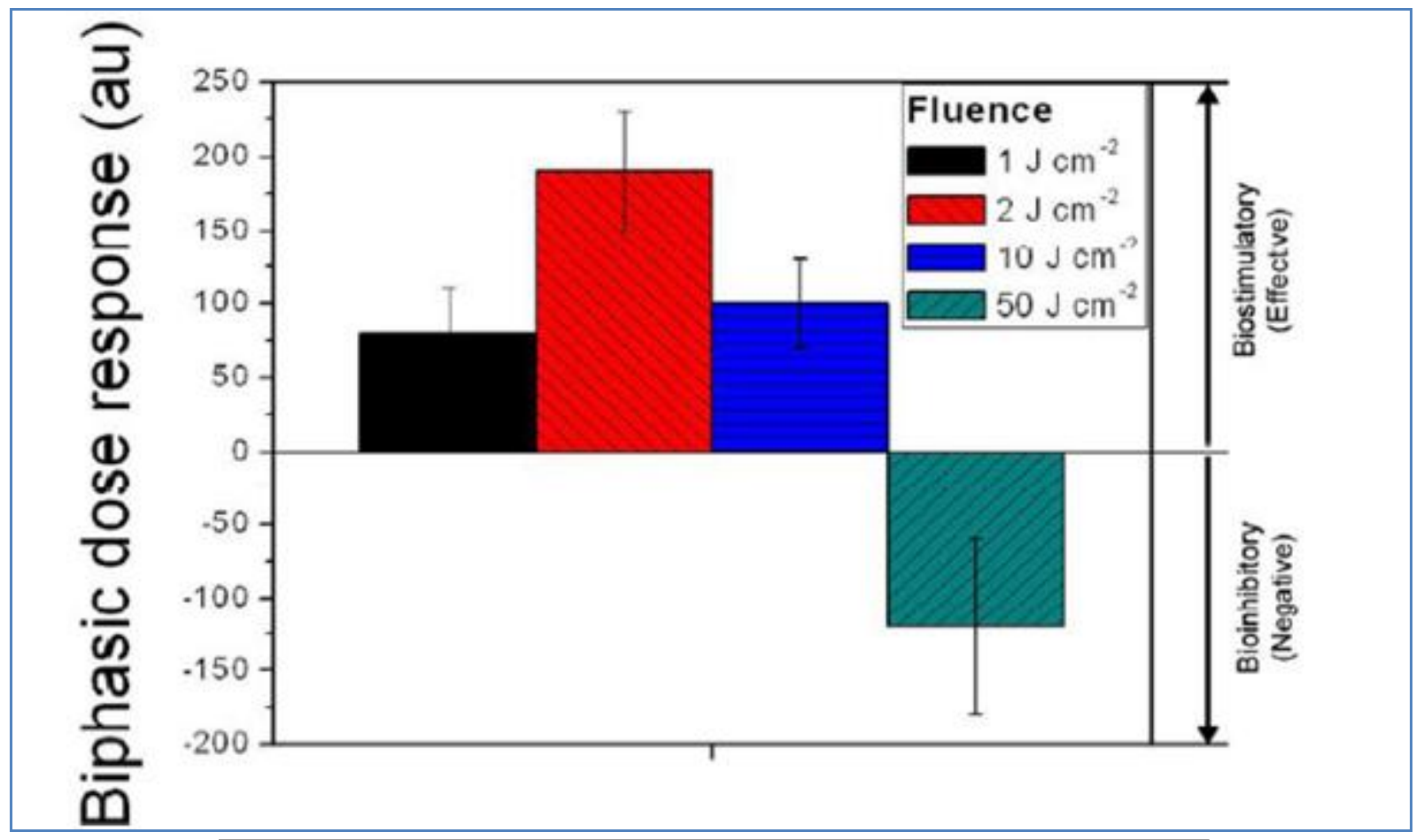

Figure 5: Biphasic dose response, based on data taken from (54)

According to Hamblin and colleagues (2009), biphasic dose response can be represented by the difference in integrated area under the curve of time tracking wound size in wounds treated with LLLT compared to no treatment controls, with a clear maximum seen at $2 \mathrm{Jcm}-2$, and a high dose of $50 \mathrm{Jcm}-2$ demonstrating a worsening of the wound healing time curve (36). In general, radiant exposures of red or NIR light as low as 3 or $5 \mathrm{~J} \mathrm{~cm}-2$ will be beneficial in-vivo, but a large dose such as 50 or $100 \mathrm{Jcm}-2$ will lose the beneficial effect and most likely will become detrimental.

Therefore, the question is no longer whether light has clinical and safety efficacy, but rather how energy from therapeutic low energy light devices (lasers or LEDs) is generated and what are the most optimal light parameters for different applications of these light sources. LLLT, as all other forms of therapeutic modalities regulated by the FDA has its active "ingredients" or irradiation parameters and therapeutic doses. 
THE IMPORTANCE OF PULSING IN LLLT (PULSE vs. CW): The efficacy of LLLT has been confirmed by several hundred randomized, double-blinded, placebo-controlled clinical trials, and this therapeutic method has been approved by the FDA, Health Canada and CE and numerous other health regulatory agencies from dozens of countries worldwide (58).

Hence, the question is no longer whether LLLT has clinical effects but rather what are the pertinent light parameters required to achieve the desired clinical effect for a particular clinical indication. Both pulsed (PW) and continuous wave (CW) modes are available in LLLT devices, which offer medical practitioners a wide range of therapeutic options, allowing them to exploit photochemical effects versus photothermal or molecular biochemical effects.

One interesting observation is the favourable effect of $905 \mathrm{~nm}$ need to explain super pulsed mode (SP) in pulse mode versus continuous wave mode ( $905 \mathrm{~nm}$ LLLT in SP mode is able to activate iNOS transcription and translation, in an acute inflammation model, demonstrating the existence of a different mechanisms of action and is dependent on the pulse duration, duty cycle and frequency of the delivered photons.

In continuous wave applications, photons interact on an individual basis with the tissue chromophores initiating a photochemical light-tissue interaction Super pulsed and pulsed sources however must execute an additional non-photochemical interaction for biostimulation to take place.

A number of well designed clinical studies have reported similar results indicating a greater efficacy of LLLT achieved with SP versus PW versus CW mode. For example sequentially pulsed optical energy was reported to be more efficacious than CW mode in stimulating collagen production in a suction blister model (59). Several other studies have also obtained a similar pattern of results.

In a wound healing study, Kymplova et al., $(60)$ used a large number of women $(n=2,436)$ to elucidate the effects of phototherapy on wound repair following surgical episiotomies (one of the most common surgical procedures in women). A pulsed laser, used in the study, emitted light (wavelength of $670 \mathrm{~nm}$, power $20 \mathrm{~mW}$ and an energy density of $2 \mathrm{~J} \mathrm{~cm}-2$ ) at various frequencies (10, 25 , and $50 \mathrm{~Hz}$ ) (the authors did not specified the pulse duration and duty cycle), and, according to the authors, promoted wound repair and healing more effectively than a CW light source (60) delivering a similar radiant exposure.

In a recent study by Brondon and colleagues (61), the photo-irradiation outcomes, following delivery of $670 \mathrm{~nm}(10 \mathrm{~mW} \mathrm{~cm}-2,5 \mathrm{~J} \mathrm{~cm}-2)$ light through a $0.025 \%$ melanin filter via continuous illumination or pulsed delivery at frequencies of 100 and $600 \mathrm{~Hz}$, were examined. Pulsing had a significantly greater stimulatory effect on cell proliferation and oxidative burst as compared to the continuous photoradiation group (61).

Taken together, these findings underline the importance of pulsing parameters in LLLT and how critical the implementation of optimal pulse duty cycle and peak power is to achieve maximal stimulation of photo bioregulatory effects in targeted tissues.

Indeed, pulsing frequency (pulse repetition rate and pulse duration) appears to hold such differential effects, as suggested by studies using different in vitro and in vivo models (62). These observations have been confirmed by Barolet D, et al., (2010). The authors have shown that certain pulsing frequencies appear to be more efficacious than others in triggering desired biological outcomes (63).

The scientific evidence continues to demonstrate that pulsed light does have biological and clinical effects that are different from those of continuous wave light. Several studies revealed that 
LLLT in a PW mode of operation is substantially better able to penetrate through the melanin and other skin barriers, supporting the hypotheses that pulsing is beneficial in reaching deep target tissue and organs.

In 2009, Lapchak P. A and Taboada L. D. reported that transcranial near infrared laser therapy (NILLLT) improves behavioural outcome following embolic strokes in embolized rabbits and clinical rating scores in acute ischemic stroke (AIS) patients.

It was proposed that mitochondrial energy production may underlie this therapeutic response to the near-infrared LLLT (NILLLT). The authors evaluated the effect of NILLLT on cortical ATP content using the rabbit small clot embolic stroke model (RSCEM), the model originally used to demonstrate NILLLT efficacy. In addition, the authors compared the effect of CW and PW illumination on ATP production in the brain tissue.

Five minutes following embolization, rabbits were exposed to 2 min of NILLLT using an 808 nm laser source, which was driven to output $262.5 \mathrm{~mW} \mathrm{~cm}-2$ either CW, or PW using $2 \mathrm{~ms}$ pulses at $100 \mathrm{~Hz}$. Three hours after embolization, the cerebral cortex was excised and processed for the measurement of ATP content using a standard luciferin-luciferase assay.

NILLLT-treated rabbits were directly compared to sham-treated embolized rabbits and naive control rabbits. Embolization decreased cortical ATP content in the ischemic cortex by $45 \%$ compared to naive rabbits, a decrease that was attenuated by CW NILLLT which resulted in a $41 \%$ increase in cortical ATP content. However, this net tissue content of ATP increase was not statistically different from either the sham-treated embolized control group or the naive control group ( $\mathrm{p}=0.1357$ and 0.0826 , respectively).

The results of the NILLLT in PW mode were much more compelling.

Following PW NILLLT, which delivered 35 times higher peak fluence rate than CW, the authors reported a 221\% ( $p=0$. 0001) increase in cortical ATP content, compared to the sham embolized group and naive control rabbits $(\mathrm{p}=0.0028)$.

The data undeniably demonstrates the benefit and the advantageous effect of pulsing in LLLT and is providing a new insight into the molecular mechanisms associated with the LLLT clinical efficacy (64).

In addition, as indicated above in association with equation 3, I stim the energy density, E/a $\left(\mathrm{Jcm}^{-2}\right)$ and the fluence rate $\left(\mathrm{Wcm}^{-2}\right)$ are important parameters. While light delivery either as $\mathrm{PW}$ or CW may not impact the energy density attainable at a given depth throughout the therapy session, the power density can be increased depending on the laser duty cycle and thus I stim can become larger than $\mathrm{I}_{0}$ at greater depth.

The attainable depth of the LLLT bioregulation increase is based on the duty cycle of the laser or the ratio of the light pulsed duration over dark period. Sinusoidal modulated light sources typically have only a duty cycle of 0.5 and hence an $\mathrm{e}^{-05}$ depth advantage of only 2 , whereas the duty cycle of a $200 \mathrm{nsec}$ pulse at $10 \mathrm{Khz}$ is 0.005 and the depth advantage can be 200 to attain the same fluence rate.

The overall body of literature on the subject has strongly indicated that clinical practitioners must not underestimate the importance of energy rate and pulse structure when using different LLLT sources. Therefore it is imperative that clinical and scientific researchers provide an explanation of how does pulsed LLLT differ from the CW LLLT on the biomolecular level, how these 
biomolecular changes are important for beneficial clinical outcomes of LLLT and what pulse parameters are critical for photobioregulation.

They should also specifically determine what is the ideal pulse repetition rate or frequency to be used, as the pulse rate may also have effects on the LLLT's mediated biological responses.

THE CONCEPT OF SUPER PULSING: Both continuous wave (CW) and continuous wave light sources that are turned on and off (pulsed light sources) cannot compare with the depth of penetration of photonic energy delivered from super pulsed lasers.

Traditionally, cold lasers deliver photonic energy from diodes that range in power from $5 \mathrm{~mW}$ to $500 \mathrm{~mW}$ per diode; otherwise known as Class $3 \mathrm{~B}$ laser devices. $200 \mathrm{~mW} / \mathrm{cm} 2$ is the highest level of irradiance allowed in the visible for a continuous wave laser (Maximum Permissible Exposure or MPE), because any greater power would result in excessive heat that could compromise the safety and the efficacy of the Low Level Laser Therapy (LLLT) treatment. However, Super Pulsed Laser can safely deliver optical energy from diodes that range in power from 10,000 $\mathrm{mW}$ to 50,000 $\mathrm{mW}$ peak power per diode.

This is paramount because the probability for a photon absorption event to occur largely depends on the photon density or fluence rate and the photon absorption cross section of the molecule where the former may be influenced by the pulse repetition rate and the peak power. In superficial tissue, where the photon density is high, it is easy to reach the necessary power density thresholds without the benefits of a super pulsed laser, in fact continuous wave lasers, pulsed lasers or even LEDs can be utilized. However, in deep tissue where the photon density is extremely low, the probability of photon absorption from individual pulsed laser speckles is extremely rare thus the probability of reaching the necessary power density thresholds, $I_{0}$, is not possible for continuous wave lasers, pulsed lasers or LEDs. However, super pulsed laser sources, through their intense pulses of light can still overcome $\mathrm{I}_{0}$ and photo chemically activate molecules at much deeper tissue depths, This effect may also explain why head-to-head comparisons between lasers and LEDs in deep tissue seem to be in favour of lasers and super pulsed lasers in particular (65).

Super pulsing allows for deeper penetration than an equivalent light source of the same wavelength that is not super pulsed but has the same average output power. This is primarily because short pulses can lead to a temporally higher concentration of excited chromophores at greater depth which in turn can impact the biochemistry at the cellular level; whereas, the period between pulses promotes optimization of thermal relaxation time of the target structure. This relaxation time, permits cooling between pulses to avoid accumulation of heat and to avoid detrimental effects, as well as to enhance the photochemical and photo physical light tissue reactions, leading to superior observant therapeutic outcomes, such as: a reduction in inflammation, elimination of pain and accelerated tissue healing.

Theralase's super pulsed infrared laser light $(905 \mathrm{~nm})$ is Health Canada, FDA and CE Mark certified and is in use in over 20 countries around the world. The super pulsed laser has the distinction of being one of the fastest in the world - delivering pulses at 200 billionths of a second, producing average powers of $100 \mathrm{~mW}$ and peak powers up to $50,000 \mathrm{~mW}$ per diode. The result is a higher concentration of light power, or photons density, exceeding $\mathrm{I}_{0}$ deeper into the target tissue, without the risk of burning the tissue. 
While CW and standard pulsed lasers are limited to less than 1-2 cm tissue depth for therapeutic efficient penetration, super pulsed infrared laser technology is able to show therapeutic efficacy at up to $10 \mathrm{~cm}$ into tissue and affect deeper target structures such as: bones, tendons, ligaments and cartilage.

In our clinical and scientific experience, $905 \mathrm{~nm}$ super pulsed technology has been proven to be more effective than $905 \mathrm{~nm}$ laser light treatment administered in a continuous wave mode (66).

TYPES OF PULSED LIGHT SOURCES: Five major types of pulsed lasers (or other light sources) are commonly utilized: 1) Q switched, 2) Gain-switched, 3) Mode-locked, 4) Super pulsed and 5) Chopped or gated. Each utilizes a different mechanism to generate light in a pulsed as opposed to continuous manner, and vary in terms of pulse repetition rates, energies, and durations. However the first three classes of pulsed lasers mentioned above are in general not used for LLLT; instead super pulsed (a "true" pulsed laser) or gated lasers are mainly used. Moreover, the chopped or gated light sources cannot compress available power into very short pulses, and hence are inefficient in converting electrical power into LLLT's optical power for very higher power densities.

SUPERPULSING OR A “TRUE” PULSED LLLT APPROACH: The concept of super pulsing was originally developed for the carbon dioxide laser used in high power tissue ablative procedures.

The idea was conceived that by generating relatively short pulses (few milliseconds) within the laser media more atoms can be excited than normally allowed in CW mode where heat dissipation constraints limit the maximum amounts of energy that can be stored in the lasing media. With the original carbon dioxide super pulsed lasers, the short pulses would confine the thermal energy in the tissue (by making the pulse duration less than the thermal diffusion time) reducing collateral thermal damage to normal tissue (67).

The type of laser that particularly benefited from super pulsing is the Gallium-AluminumArsenide (GaAlAs) and the Aluminum-Gallium-Indium-Phosphide (AlGaInP) diode laser. This GaAlAs laser has a wavelength in the region of 904 to $\mathrm{nm}$ and pulse duration as short as 100 to 200 nanoseconds can be achieved.

Another semiconductor laser amenable to super pulsing is the Aluminum-Gallium-IndiumPhosphide (AlGaInP) diode laser. It emits light at a similar wavelength $(905 \mathrm{~nm})$ as the GaAlAs diode laser, producing very brief pulses (100 to 200 nanoseconds) of high frequencies (in the range of kilohertz). These pulses are of very high peak powers $(1-50 \mathrm{~W})$ and an average power of $150 \mathrm{~mW}$ to $650 \mathrm{~mW}$.

Theoretically, super pulsed GaAlAs and AlGaInP lasers allow $\mathrm{I}_{0}$ to be exceeded at greater depth without the unwelcome effects of CW (such as thermal injury), as well as allowing for shorter treatment times, and, hence, offer advantageous dose delivery and clinical benefits without the undesirable adverse effects, such as including tissue damage or unfavourable biphasic effects.

Super pulsed lasers produce very powerful, pulsed peak power in the Watt range, but the duration of the peak is typically only 100 to 200 nanoseconds. A GaAlAs laser presenting a peak power of $10 \mathrm{~W}$ typically has an average output of $20 \mathrm{~mW}$ due to its very low duty cycle.

The other major class of pulsed light sources used in LLLT are not true pulsed lasers, but simply CW lasers (usually diode lasers) that have a pulsed power supply generated by a laser driver containing an often sinusoidal pulse generator. This technology is described as "chopped" or "gated" 
or "pseudo". It is also equally feasible to use pulse generator technology to pulse LEDs or LED arrays (68) albeit the ability to produce 100s of nsec pulses has not been demonstrated.

THE BIOLOGICAL IMPORTANCE OF “TRUE” PULSING IN LLLT: A “true' pulsed or super pulsed light offers numerous potential benefits. Because there are "quench periods" (pulse OFF times) following the pulse $\mathrm{ON}$ times true, pulsed lasers or super pulsed lasers generate less tissue heating. In instances where it is desirable to deliver light to deeper tissues increased powers are needed to provide adequate power density at the target tissue, so I stim > I o. This increased power can cause a transient tissue heating, particularly at specific biological targets (as discussed above in the mitochondrial mechanisms of LLLT) and in this instance pulsed light could be very important. Whereas CW or PW-generated by not "true" pulsed lasers (including "chopped" or "gated" or "pseudo" technology), particularly emitted by non-coherent (non laser light sources), causes an undesirable increase in temperature of the intervening and target tissues or organ.

Moreover, a "true" pulsed light that is used in LLLT has been shown to cause no measurable change in the temperature of the irradiated area for the same delivered energy density as other nonpulsed therapeutic light sources (58).

In 2006, Ilic S., et al., found that pulsed light (peak power densities of $750 \mathrm{mWcm}-2$ ) administered for 120 seconds produced no neurological or tissue damage, whereas an equal power density delivered by CW (for the same number of seconds) caused marked neurological deficits (69).

Aside from safety advantages true, pulsed or super pulsed light might simply be more effective than CW. The "quench period" (pulse OFF times) reduces tissue heating; thereby, allowing the use of potentially much higher peak power densities than those that could be safely used in CW. The higher power densities can change the reaction balances in favour of a positive signaling effect or through the creation of thermal micro gradients changing the analyte exchange across cellular and subcellular membranes. For example, when CW power densities at the skin use equal or higher than $2 \mathrm{Wcm}-2$, doubling the CW power density would only marginally increase the treatment depth while potentially significantly increasing the risk of thermal damage; in contrast, peak powers of equal or higher than $5 \mathrm{Wcm}-2$ pulsed using appropriate $\mathrm{ON}$ and OFF times might produce little, or no tissue heating. The higher pulsed peak powers can avoid tissue heating problems and improve the fluence rate at deep tissues, achieving greater treatment depths and hence better, overall therapeutic efficacy $(40 ; 63)$.

There may be other biological reasons for the improved efficacy of pulsed light (PW) over CW. According to Hashmi J. T., et al., the majority of the pulsed light sources used for LLLT have frequencies in the $2.5-10,000 \mathrm{~Hz}$ range and pulse durations are commonly in the range of a few milliseconds. This observation suggests that if there is a biological explanation of the improved effects of pulsed light it is either due to some fundamental frequency that exists in biological systems in the range of tens to hundreds of $\mathrm{Hz}$, or alternatively due to some biological process that has a time scale of a few milliseconds (58). Indeed, the possibility of resonance occurring between the frequency of the light pulses and the neuronal electromagnetic frequency may in some way explain a number of the beneficial results with LLLT using true pulsed light (70).

In addition, there are several lines of evidence that ion channels are involved in the subcellular effects of LLLT, playing the critical role in the intercellular signaling induced in tissues by LLLT (71). 
Some channels permit the passage of ions based solely on whether their charge is of positive (cationic) or negative (anionic) while others are selective for specific species of ion, such as sodium or potassium. These ions move through the channel pore single file nearly as quickly as the ions move through free fluid. In some ion channels, passage through the pore is governed by a "gate, " which may be opened or closed by chemical or electrical signals, temperature, or mechanical force, depending on the variety of channel. Ion channels are especially prominent components of the nervous system.

Voltage activated ion channels underlie the nerve impulse while transmitted, activated or ligand gated channels mediate conduction across the synapses. There is a large body of literature on the kinetics of various classes of ion channels but in broad summary it can be claimed that the time scale or kinetics for opening and closing of ion channels is of the order of a few milliseconds.

For instance, there are several reports indicating that the diverse types of ion channel receptors have kinetics with timescales that are comparable with the super pulsing technology (72; 73), as well potassium and calcium ion channels in the mitochondria and the sarcolemma may be involved in the cellular response to $\operatorname{LLLT}(74 ; 75)$.

Several authors have also suggested that there is the possibility that one cellular mechanism of action of LLLT is the photo dissociation of nitric oxide from a protein binding site (heme or copper center) such as those found in cytochrome c oxidase. If this process occurs it is likely that the NO would rebind to the same site even in the presence of continuous light. Therefore if the light was pulsed multiple photo dissociation events could occur, while in CW mode the number of dissociations may be much smaller (58).

THE LIGHT PENETRATION DEPTH: The most important parameter that governs the depth of penetration of laser light into tissue is wavelength. When light at a specific wavelength interacts with particular tissue, it is either absorbed or scattered in various proportions depending on the optical properties of the tissue. The tissue characteristics are important for LLLT and all other forms of phototherapy and medical laser applications, in order to understand the interaction mechanisms between light and tissue. Knowledge concerning light transport in tissue and hence the composition of tissue is essential for proper light dosimetry. In general, both the absorption ( $\mu$ a)and scattering $(\mu s)$ coefficients of living tissues are higher at lower wavelengths, so near-infrared light (up to about $950 \mathrm{~nm}$ ) penetrates more deeply that visible and UV light. It is often claimed that pulsed lasers penetrate more deeply into tissue than CW lasers with the same average power density (76).

Many applications of LLLT may require deep penetration of the light energy, such as deep treated conditions like: lower back, neck, and hip joint pain and inflammation. Therefore, if the power densities need to be greater than a few $\mathrm{mWcm}-2$ and are required to be delivered safely to target tissues $>5 \mathrm{~cm}$ below the skin surface, the effective treatment can only be achieved by using super pulsed lasers (58).

THE USE OF COMBINED LASERS (CW + PW) IN LLLT: Scientific and clinical data suggest that a relatively high fluence (the light energy density delivered to the target tissue) is necessary to attenuate pain, whereas a lower fluence decreases inflammation. If this is indeed the case, for a number of clinical conditions, including musculoskeletal applications, achieving higher doses at the level of the target tissue may not be ideal. 


\section{REVIEW ARTICLE}

It has also been postulated that successful LLLT treatments of arthritic joints bring benefit not by reaching the deep target tissue directly but by inhibiting superficial nociceptors. In other words, the therapy brings relief primarily by attenuating pain perception, as opposed to reduction of deep tissue inflammation (58).

It becomes evident that the use of a prescribed dose at the tissue surface is insufficient to reach a desired target dose at depth, as the tissue thickness to the target differs between patients, as do the tissue attenuation coefficients which are based on tissue compositions. Hence, new technology is being developed to, optimize these variables and develop a smart) technology that enables the determination of the power density, Istim and the fluence at the target tissue's depth.

These scientific reports, as well as, observations on the complementary biological actions of the far-red and near-infrared irradiation have led to the beginning of the research and development of a unique class of platform technologies that combine (CW + PW and SP) LLLT approaches; and after FDA and other health regulatory agencies approvals in Canada, Europe, Latin America and in Asia, to a successful commercialization of the new and effective phototherapeutic medical devices.

The latest medical device technology in the field employs visible red (CW) and near infrared super pulse (SP) proprietary technology to comprise a unique and clinically validated array of multiple probe lasers. As a result of this synchronizing therapeutic approach, benefits offered by this new technology includes: 1) delivery of a well-defined, particular dose of coherent photon energy per treatment given to a patient and 2) implementation of cooperative stimulation of the proximal and distal therapeutic mechanisms, in tissues, to induce bioregulatory responses that mitigate inflammation and pain, as well as, the ability to accelerate the healing in the disease in affected areas and assist in maintaining remission, particularly through ${ }_{(3)}$ delivering energy at Istim > Io to the required depth. Up until now, in the peer-reviewed literature, there has been remarkably little information available about any laser technology that encompasses these concepts.

In Gigo-Benato's study, CW or PW stand alone was compared to combined laser. In short, the combined laser was found to be more effective in stimulating nerve regeneration, than either CW or PW alone (77).

The two other studies used a combined laser (CW and PW) to administer laser acupuncture, along with Transcutaneous Electrical Nerve Stimulation (TENS), to patients with symptoms of pain. Naeser et al., administered this "triple therapy" to patients suffering from carpal tunnel syndrome (CTS) (78).

Eleven patients with mild-to-moderate symptoms of CTS were selected to participate in the trial. All participants were previously treated by various methods, but had failed to respond to standard medical or surgical treatment regimens. Subjects were divided into two groups, one of which received sham irradiation and the other received a combined treatment of LLLT (CW and PW) and TENS. As compared to controls, the treated group experienced statistically significant improvement and remained stable for 1 to 3 years.

The results of this study indicate the therapeutic advances of combined LLLT in the treatment of CTS. Ceccherelli et al., (1989) administered laser acupuncture to patients suffering from myofascial pain (79). In this double blinded placebo controlled trial, patients received either the same "triple therapy" as in the Naeser et al. study (CW, PW, and TENS) or sham irradiation, every other day over the course of 24 days. Results were encouraging, with the treatment group experiencing a significant improvement in symptoms, both immediately after the treatment regimen 
and at a 3-month follow up visit. In both preceding studies, the combined regimen of CW, PW, and TENS was compared to untreated controls, and found to be effective (78). However, neither study compared CW and PW or administered CW, PW, or TENS individually. Therefore, it is impossible to determine whether stand alone the combination of CW and PW light would have produced similar results, or if the used combined LLLT regimen along with TENS was more effective.

As described above, the therapeutic optical windows (660 nm and $905 \mathrm{~nm}$ ) utilized by The latest LLLT technologies are correspond to the absorption and the action spectra optical windows of the key mitochondria chromophores, such as cytochrome c oxidase and cellular membrane lipids. Moreover, it is apparent that $660 \mathrm{~nm}$ and $905 \mathrm{~nm}$ light have an impact on the mitochondrial chromophores via independent mechanisms and hence the combination of $660 \mathrm{~nm}$ and $905 \mathrm{~nm}$ light is considered highly probable to have an additive biologic effect compared to individual wavelengths. This biologic effect is amplified to achieve the effective therapeutic outcomes via implementation of the latest LLLT technology cooperative CW and PW stimulation system that target the proximal and distal therapeutic mechanisms, in tissues, to induce bioregulatory responses that effectively modulate local and systemic pathologic manifestations in the LLLT treated patients.

Latest LLLT technology provides significant clinical symptoms relief and a quality of life improvement in patients with osteoarthritis and untreated injuries to muscles, ligaments and tendons that were enrolled into the study based on the Philadelphia Panel Classification, 2001 (80).

Considering the multifactorial etiopathogenesis of the majority diseases, it is likely that the efficacy of LLLT is determent by optimal sets of therapeutic wavelengths, which should be optimized based on the optical properties of treated tissues and regiments of light delivery targeting particular chromophores at specific tissue depth.

\section{BIBLIOGRAPHY:}

1. Comparison of four non-invasive rewarming methods for mild hypothermia. Daanen, H. A. and Van de Linde, FJ. 12, s. l. Aviat Space Environ Med, 1992, Vol. 63, pp. 1070-1076.

2. Transcutaneous electrostimulation for osteoarthritis of the knee. Rutjes, AW, et al. 4, Oct 2009, Cochrane Database Syst Rev, Vol. 7.

3. Tennis elbow. Clinical Evidence. Bisset, L, Coombes, B and Vicenzino, B. [Online] june 27, 2011. [Cited: June 27, 2009.] http://clinicalevidence. bmj. com/ceweb/index. jsp.

4. Safety of Lase Products user's guide. s. l. Commission, International Electro technical. IEC International Electro technical Commission, 2004. TR 60825-14.

5. Low power laser treatment in patients with knee osteoarthritis. Tascioglu, F, et al. 17-18, May 1, 2004, Swiss Med Wkly, Vol. 134, pp. 254-258.

6. In vivo effects of low level laser therapy on inducible nitric oxide synthase. Moriyama Y, Nguyen J, Akens M, Moriyama EH, Lilge L. 3, March 2009, Lasers Surg Med, Vol. 41, pp. 227231.

7. Photo dissociation of cytochrome c oxidase-nitric oxide complexes. Gorren AC, Van Gelder BF, Wever R. 1988, Ann N Y Acad Sci, Vol. 550, pp. 139-149.

8. Mitochondrial nitric oxide synthase: current concepts and controversies. Lacza Z, Pankotai E, Busija DW. 14, Jan 2009, Front Biosci, Vol. 1, pp. 4436-4443.

9. Nitric oxide and mitochondria. GC. Brown. 12, Jan 2007, Front Biosci, Vol. 1, pp. 1024-1033. 


\section{REVIEW ARTICLE}

10. Redox signaling (cross-talk) from and to mitochondria involves mitochondrial pores and reactive oxygen species. A, Daiber. 6-7, Jul 2010, Biochim Biophys Acta, Vol. 1797, pp. 897906.

\section{AUTHORS:}

1. Samith Ahmed

2. Gregory Bewsh

3. Shankaranarayana Bhat

4. Ramesh Babu

\section{PARTICULARS OF CONTRIBUTORS:}

1. Consultant Physician, Department of Alternative Medicine, Sree Sanjeevini Solutions.

2. Research Manager, Department of Alternative Medicine, Sree Sanjeevini Solutions.

3. Branch Manager, Department of Alternative Medicine, Sree Sanjeevini Solutions.

4. CEO, Department of Alternative Medicine, Sree Sanjeevini Solutions.

\section{NAME ADDRESS EMAIL ID OF THE} CORRESPONDING AUTHOR:

Dr. Samith Ahmed, Sree sanjeevini cold laser cafeNo 316, 3rd floor Sree Krupa 15th cross opp to Karur vysya bank

Sampige road, Malleswarm

Bangalore-560003

Email-samith_17@yahoo.com

Date of Submission: 03/09/2013.

Date of Peer Review: 07/09/2013.

Date of Acceptance: 20/09/2013.

Date of Publishing: 24/09/2013 